\title{
How to Use Simulation for Heavy Oil / Tar Mat Recovery Methods?
}

\section{Paul Boerrigter, Shell}

Heavy oil displacement processes pose severe challenges to reservoir simulators. Not only the physics is complex (thermal/steam, miscible effects, chemical reactions), also the mobility of displacing fluids is usually much higher than the mobility of the heavy oil, leading to displacement processes that are inherently unstable. Geological small scale inhomogeneities strongly influence these instable displacement patterns, often leading to a lack of conformance, both laterally and vertically, in reservoir sweep. In reservoir simulators, instable displacement shows a strong sensitivity to grid structures. To minimize these gridorientation effects special gridding systems (9-point schemes as alternative to 5-point schemes) are already for a long time in use.

This presentation is intended to be an introduction into a discussion on how simulation is best used to describe Heavy Oil / Tar Mat recovery techniques. It will be of interest to hear from the different participants how they deal with the issues mentioned above and how they feel about simulator capability to describe new/improved recovery techniques

The presentation will discuss, as introduction to the topic, some recent developments:

- The effect of improved gridding schemes like dynamic local grid refinement on grid orientation effects.

- Modeling requirements for improved recovery techniques. An example is the use of solvents co-injected with steam. Condensing solvent will dilute the bitumen, making it easier to recover. Modeling requires fine grid at steam/solvent fronts and adequate viscosity mixing rules for dead and live crudes. The viscosity rules are also relevant for reservoirs with a strong compositional grading, as is common for reservoirs with tar mats. 